\title{
Persistent Current Effects in BSCCO Common Coil Dipoles
}

\author{
William B. Sampson, Arup K. Ghosh, John P. Cozzolino \\ Michael A. Harrison and Peter J. Wanderer
}

\begin{abstract}
A series of one-meter long racetrack-shaped windings has been fabricated from BSCCO tape conductors obtained from four manufacturers. Two coils were built from each conductor type and tested in the "common coil" dipole configuration in liquid helium. The effect of the remnant magnetization currents was determined by measuring the residual dipole and sextupole fields after cycling the magnets to progressively higher currents. Two coil sets have been measured and the results are compared to those obtained from a $\mathrm{Nb}_{3} \mathrm{Sn}$ ribbon magnet of the same geometry.
\end{abstract}

Index Terms-Common Coil Magnets, remnant fields, HTS BSSCO-2223 tape conductor

\section{NTRODUCTION}

Common coil dipoles in which the windings are powered in such a way that regions of opposite polarity occur in the same magnet are ideally suited to collider applications [1,2]. The fact that the minimum-bending radius of the superconductor can be made quite large in such a design means that relatively brittle materials can be used in their construction. High Temperature Superconductors (HTS) in general have limited flexibility so that the common coil approach provides a natural way of exploiting the potential of these materials. This combination of a novel magnet design and a new type of conductor makes it difficult to estimate the effect of persistent currents on the magnetic field distribution. Direct measurements were made on the residual fields of two magnets made from BSCCO-2223 tape produced by different suppliers, Intermagnetic General Corporation (IGC) and American Superconductor Corporation (ASC). A common coil dipole wound from $\mathrm{Nb}_{3} \mathrm{Sn}$ tape was also measured. Additional magnets in this series will be tested for remnant fields as they become available.

\section{MAGNET CONSTRUCTION DETAILS}

The coils were wound on a flat table with conductor and insulation feeding from separate pay-off spools. The tension was controlled using torque motors with most of the force applied to the Kapton insulating ribbon. As each half turn was positioned it was held in place by a clamping bar while

Manuscript received September 18, 2000.

This work was supported by the U.S. Department of Energy under contract No. DE-AC02-98CH10886.

The authors are with Brookhaven national Laboratory, Upton, NY 11973 (telcphone : 631-344-2036, e-mail: ghosh1@bnl.gov).

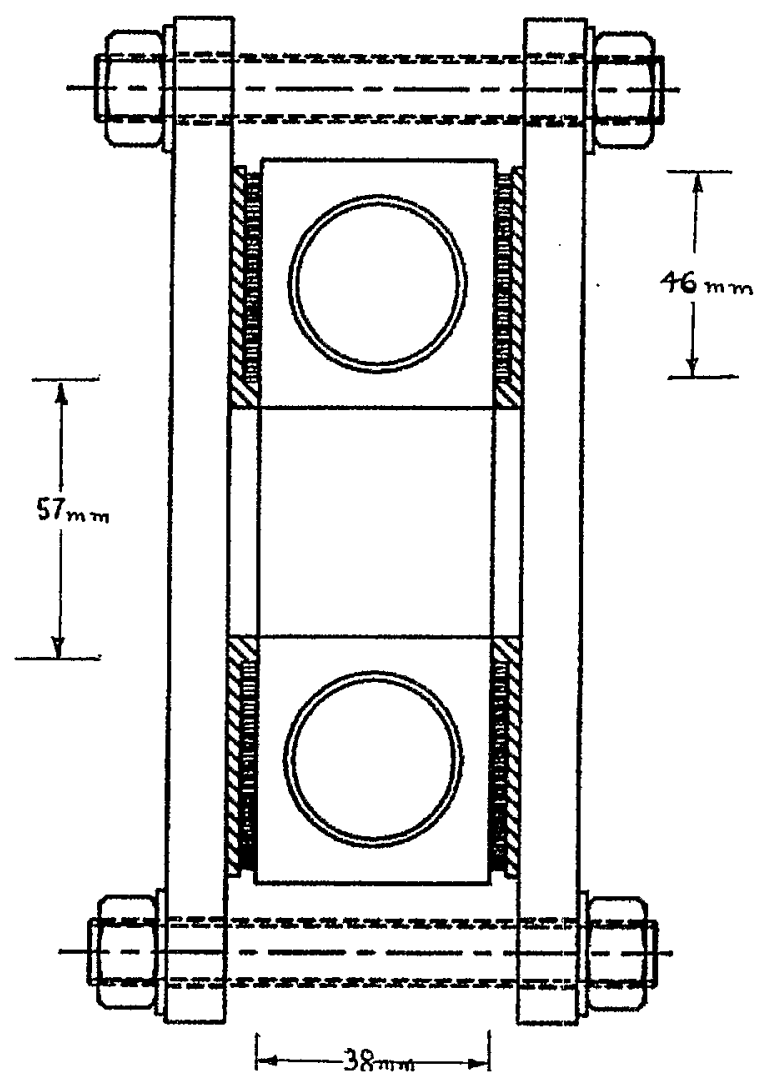

Fig. 1. Test configuration of the racetrack coils in "common-coil" form.

the next half turn was wound. Joints between sections of ribbon were formed as needed in the end regions by overlap soldering. Voltage taps were provided by inserting thin copper foils into the coils at the beginning and end of each conductor length and on either side of any unusual sections that appeared during winding. Finished coils were coated with epoxy and heat cured to form quite rigid and easy to handle structures. A common coil magnet was assembled from two racetrack coils in an aluminum fixture clamped by brass bolts as shown in Fig. 1. The separation between coils is $38 \mathrm{~mm}$ and an access hole of $32 \mathrm{~mm}$ is available for the magnetic measuring probe. Four sets of BSCCO-2223 coils are being fabricated with conductor from different sources. The windings have the same overall dimensions except for the thickness, which is determined by the width of the ribbon. The number of turns in each set varies due to conductor thickness. Details of the windings are summarized in Table 1. 
TABLE I

RACETRACK COIL PARAMETERS

\begin{tabular}{cccc}
\hline $\begin{array}{c}\text { Conductor } \\
\text { Type }\end{array}$ & Dimensions, mm & Turns/Coil & Status \\
\hline $\mathrm{Nb}_{3} \mathrm{Sn}$ & $0.2 \times 3.2$ & 168 & Tested \\
$\mathrm{IGC}$ & $0.25 \times 3.3$ & 147 & Tested \\
$\mathrm{ASC}$ & $0.18 \times 3.1$ & 221 & Tested \\
$\mathrm{NST}$ & $0.20 \times 3.2$ & 220 & $\begin{array}{c}\text { Under } \\
\text { construction } \\
\text { Under }\end{array}$ \\
$\mathrm{VAC}$ & $0.23 \times 3.4$ & 170 & $\begin{array}{c}\text { Under } \\
\text { construction }\end{array}$ \\
\hline
\end{tabular}

After testing in their self field in the fixture shown in Fig. 1 the coils will be measured as inserts in a high field NbTi test magnet.[3]

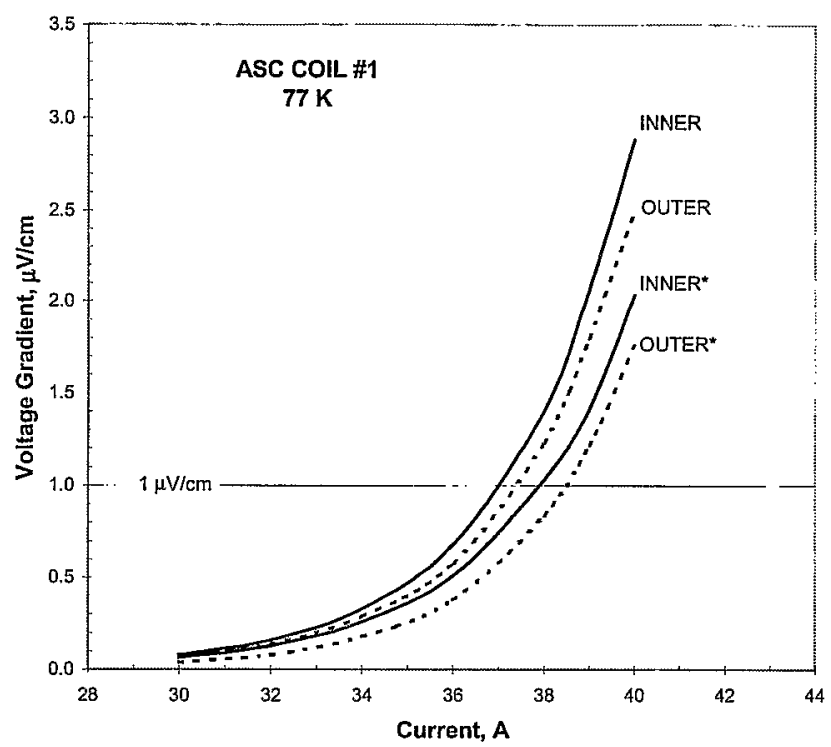

Fig. 2. The average voltage gradient across the inner and outer sections of ASC Coil \# 1 as a function of current measurcd in liquid nitrogen. The curves marked with a star are for the case where only half the magnet is powered.

\section{RACETRACK COIL PERFORMANCE}

The first pair of HTS racetrack coils was made from 2223 ribbon produced by IGC. This conductor was received over two years ago and had quite low current carrying ability. The performance of this set of coils was limited to 110 amps at $4.2 \mathrm{~K}$. The ASC windings were a significant improvement and could be operated at currents as high as 300 amps despite the smaller cross sectional area of the conductor. Each of the ASC coils was wound from two $210 \mathrm{~m}$ lengths of conductor. In Fig. 2 the $77 \mathrm{~K}$ voltage-current characteristics are shown for the inner and outer sections of Coil \# 1 when powered as either a single winding or part of a common coil magnet. It is obvious from this diagram that all sections of the winding exhibit approximately the same effective resistivity independent of their position in the coil and the local magnetic field distribution. The current at which the average voltage gradient along the conductor equals $1 \mu \mathrm{V} / \mathrm{cm}$ is the same to within about 1 amp for the whole magnet or any individual section and is approximately one half of the zero field value. The situation in liquid helium is similar as shown in Fig. 3 where the voltage gradient characteristics of the whole magnet are compared to the two sections of one of the racetrack coils. Again at an average gradient of $1 \mu \mathrm{V} / \mathrm{cm}$ the current is almost the same but the voltage gradient in the outer section is increasing more rapidly than the inner and ultimately it is this section which limits the magnet performance. At lower effective resistance most of the voltage appears to be across the inner turns but the smaller gradients are more difficult to measure accurately due to the large inductive voltages arising from power supply ripple.

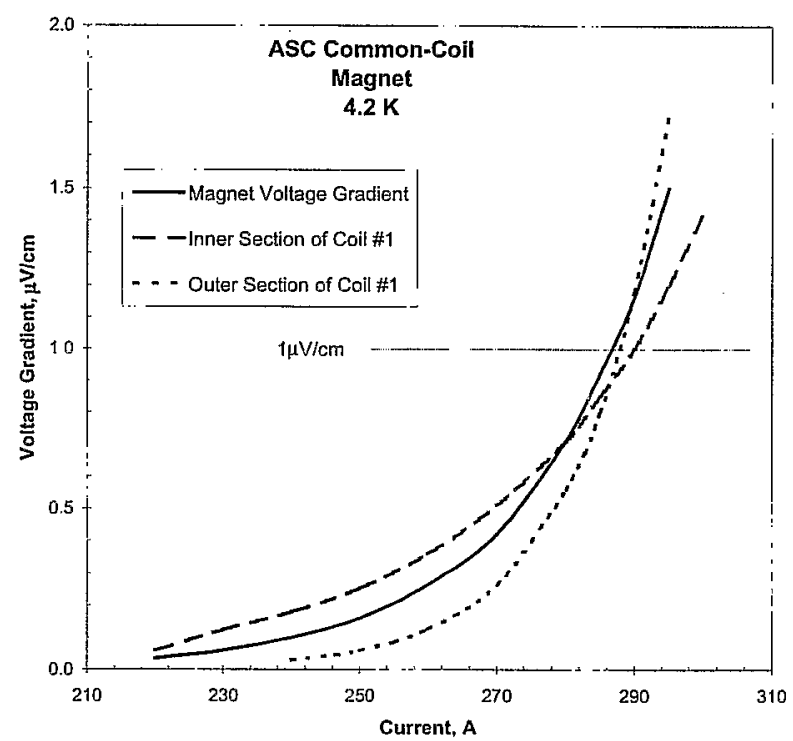

Fig. 3. The average voltage gradient across the whole magnet compared with the inner and outer sections of Coil \# 1 measured in liquid helium.

\section{MAGNETIC MEASUREMENTS}

The effect of conductor magnetization was characterized by measuring the trapped field at zero current after energizing the magnet to progressively higher currents. The remnant dipole and sextupole fields were measured since they are by far the largest and most important components. The technique used employs Hall probes mounted in a G-10 cylinder, which can be rotated in one of the magnet apertures. Fig. 4 shows the arrangement of the three Siemens type SBV 604 Hall plates. As the cylinder is rotated each unit produces a signal proportional to the tangential component of the field. By reading the values at discrete angular increments and performing a fourier analysis, the various harmonics can be resolved. If the three probes are carefully adjusted to the same sensitivity and connected in series the output is proportional to the sextupole component only and the array has three times the sensitivity of a single plate. An example is shown in Fig. 5 where the output of the three Hall array is plotted against angle for a sextupole field of about 2 gauss in 
electron beam, which is velocity matched to the ion beam, is then introduced into the $1 \mathrm{~T}$ cooling solenoid, overlapping the ion beam. Since the ion beam is much longer than the electron beam, the phase of the electron beam will be modulated in order to cool the required longitudinal extent of the ion beam. Other modulations (in energy and radial coordinates) may be introduced to shape the ion beam in phase-space. Emerging from the $30 \mathrm{~m}$ long cooling solenoid, the electron beam will be separated from the ion beam, rebunched (to match the linac acceptance) and decelerated to recover its energy. The beam will be dumped at about $5 \mathrm{MeV}$.

There are a few straight sections in RHIC where the electron cooler may be introduced. We are considering a placement next to IP4 of RHIC, in the straight section between Q3 and Q4, which can accept the $30 \mathrm{~m}$ long solenoids. The electron accelerators will be placed outside the RHIC tunnel.

There are a number of issues to be investigated. The brightness of the electron source is one. We have to produce a high-brightness beam with a high chargeper-bunch in a CW operation. A photoinjector looks promising, but so does a DC gun based system developed at BINP. At this time we are proceeding with both options open. Another one is the high-current energy recovery linac, requiring a current of about 100 $\mathrm{mA}$, or 20 times higher than what has been demonstrated so far. The $1 \mathrm{~T}, 30 \mathrm{~m}$ long ultra-high precision solenoid is another challenge. The required precision is of the order of the ions' angular spread, $\Delta \theta$, given by:

$$
\Delta \theta:=\sqrt{\frac{\text { sni }}{\beta \cdot \gamma \cdot \beta \operatorname{cool}}}
$$

where $\varepsilon_{\mathrm{mi}}$ is the ions' normalized emittance and $\beta_{\text {cool }}$ is the beta function in the cooler solenoid. In our case $\Delta \theta$ is about $10^{-5}$.

\section{ANTICIPATED PERFORMANCE}

We assume a $100 \mathrm{GeV} / \mathrm{u}$ gold beams in the collider, with either 60 (RHIC) or 120 bunches (RHIC II) stored in each ring. The initial emittance of $15 \mathrm{~mm} \mathrm{mrad}$ (normalized, 95\% emittance) will be cooled to about 6 or less. The bunch population is assumed to be $10^{9}$. The IP beta function is $2 \mathrm{~m}$ (RHIC) or $1 \mathrm{~m}$ (RHIC II).

The $100 \mathrm{GeV} / \mathrm{u}$ gold beam will be cooled only very slightly, to increase its peak luminosity. The beam should not be cooled too far for two reasons. First, the beam-beam parameter may exceed its maximum stable value estimated at 0.004 . Second, as discussed in section 2.2 , increasing the luminosity too much just leads to a rapid disintegration of the beam in the IP and to a variable luminosity as well as short store times. This can be seen in the figure below, showing the luminosity as a function of time for various cooling rates, using $10^{10}, 3 \times 10^{10}$ and $10^{11}$ electrons per bunch. The luminosity with no cooling is also plotted, showing how IBS causes a drop in luminosity due to beam loss and emittance increase. Vigorous cooling can lead to a rapid increase, followed by a rapid decline in the instantaneous luminosity. Naturally the cooling can be adjusted to maintain a constant luminosity (at a lower value than the peaks) over the store period to optimize the collider performance.

The luminosity increase that we expect the RHIC II upgrade to deliver is about 40 , of which about a factor of 4 is planned from beta function reduction and increase in the number of bunches, a factor of 10 is anticipated to come from the electron cooling.

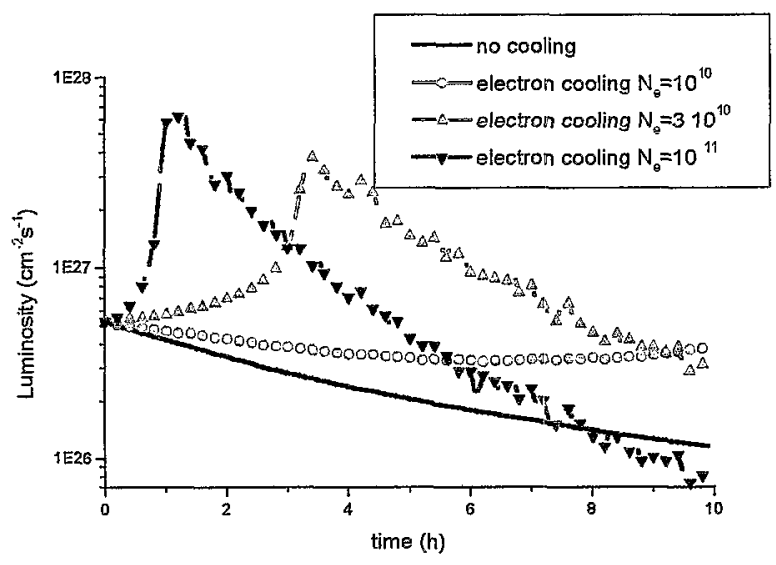

Figure 2. Luminosity as a function of time for a few values of the electron charge per bunch [1].

\section{REFERENCES}

[1] Electron Cooling for RHIC Design Report, V.V. Parkhomchuk and I. Ben-Zvi, Principal Investigators, 2001.

[2] M. Bell, J.S. Bell Particle Accelerator 12 p.49 (1982)

[3] G. Gwinner et al., Nuclear Instruments and Methods in Physics Research A441 p.183-190 (2000)).

[4] Baltz et al., Phys. Rev. E 54, 4233 (1996)

[5] A. Burov et al., Phys. Rev. ST Accel. Beams 3, $094002(2000)$ 


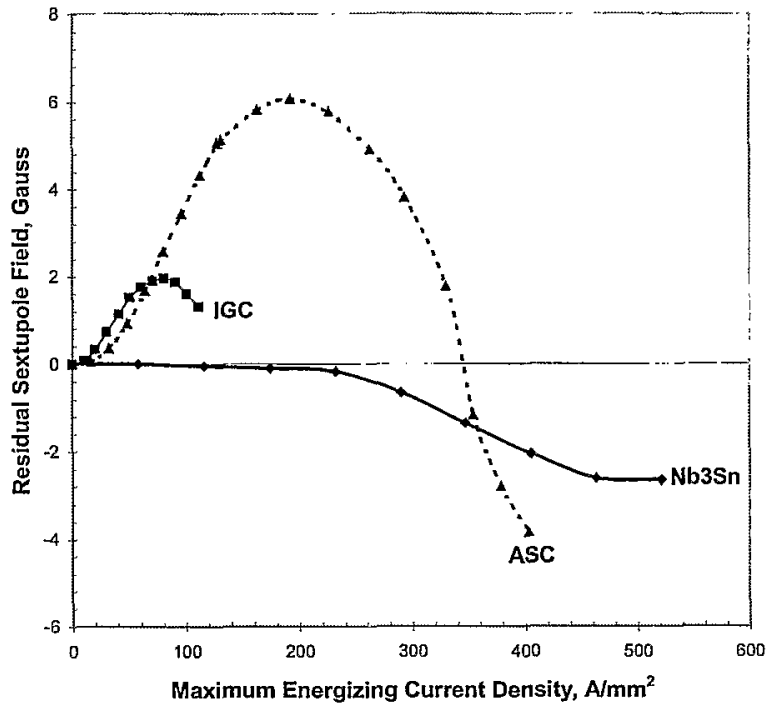

Fig. 7. The remnant sextupole field as a function of the peak current density for all three common coil magnets.

\section{ACKNOWLEDGEMENT}

The authors would like to thank A. O. Werner for fabricating the coils and test equipment and J. A. D'Ambra for analysis of the residual field patterns.

\section{REFERENCES}

1) R. Gupta, et al, "Common Coil Magnet Program at BNL", these proceedings.

2) W.B.Sampson and A.K. Ghosh "The Performance of BSCCO Racetrack Coils at 4.2K", IEEE. Trans. On Applied

Superconductivity, vol. 9, No.2, pp. 289, 1999

3) A.K. Ghosh et al., "A Common Coil Magnet for Testing High Field Superconductors", Prooceedings of the 1999 Particle Accelerator Conference 